Cahiers de recherches médiévales

\title{
Présence de la littérature française du Moyen Âge dans le cinéma français
}

\section{François Amy de la Bretèque}

\section{(2) OpenEdition}

Édition électronique

URL : https://journals.openedition.org/crm/2499

DOI : $10.4000 / \mathrm{crm} .2499$

ISSN : 1955-2424

Éditeur

Honoré Champion

\section{Édition imprimée}

Date de publication : 12 décembre 1996

Pagination : 155-165

ISSN : $1272-9752$

\section{Référence électronique}

François Amy de la Bretèque, « Présence de la littérature française du Moyen Âge dans le cinéma

français ", Cahiers de recherches médiévales [En ligne], 2 | 1996, mis en ligne le 04 février 2008, consulté le 15 décembre 2022. URL : http://journals.openedition.org/crm/2499; DOI : https://doi.org/10.4000/ crm.2499 


\section{RM}

\section{Présence de la littérature française du Moyen Age dans le cinéma français}

Le Moyen Age constitue-t-il en France une source d'inspiration nationale? Plus précisément, la littérature médiévale représente-t-elle chez nous un ensemble intertextuel encore actif dans les créations modemes? Un examen panoramique de la production cinématographique française au long du premier siècle peut fournir des éléments de réponse à ces questions. Il permettra de réviser certaines idées reçues. On a tendance à penser que le cinéma français s'est assez peu intéressé à cette période de notre histoire, et de notre littérature, contrairement à d'autres: les Anglo-saxons en paraissent culturellement plus imprégnés, et nos cinéastes semblent avoir préféré d'autres époques (les siècles classiques ou le $\mathrm{XIX}^{9}$ ); on leur prête surtout un penchant pour les études de mours contemporaines. Impression qu'a renforcé l'ostracisme qui frappa le film en costumes à une certaine époque, notamment au temps de la Nouvelle Vague.' Cette timidité, nous, Français, aimons l'imputer à un respect du passé plus grand. Aux Américains le romanesque, à nous la fidélité.

Un examen un peu attentif de la production française oblige à corriger cette impression. La curiosité des cinéastes français pour les œuvres littéraires que le Moyen Age nous a léguées a été plus répandue que ce qu'on pense. Elle commence plus tôt qu'on ne le croit. Certes, le bilan numérique n'est pas très impressionnant, mais il atteste que la littérature médiévale a constitué une source d'inspiration pour les scénaristes et les réalisateurs, à certaines époques en tout cas.

On s'intéressera ici surtout aux films de la première moitié du siècle. Pourquoi? Parce que cette production est largement ignorée, d'abord. Or, elle fait preuve d'une attention au patrimoine médiéval bien supérieure à ce que l'on croit. Il est étonnant de relever que cette curiosité restera ensuite longtemps en friches. La deuxième raison de nous attarder sur cette période, c'est que les films plus récents et plus connus, que l'on salue en général comme une redécouverte du Moyen Age (le Lancelot de Bresson ou le Perceval de Rohmer) prennent leur place au sein d'une longue durée. Ils ne sont pas des aérolithes isolés, mais retrouvent une tradition interrompue, fût-ce pour la détourner complètement.

Pour conduire cette rapide analyse, j'ai classé arbitrairement la matière littéraire en trois grandes rubriques. La première regroupe les formes littéraires que le cinéma a largement négligées, et qui constituent pourtant les plus anciennes formes narratives de l'Occident. La seconde représente le bloc le plus important, celui qui dérive des romans communément baptisés "de chevalerie». On fera en troisième lieu une place aux traditions ni chevaleresque, ni courtoise (celle que les manuels traditionnels baptisaient «littérature bourgeoise»), dont la trace dans le cinéma peut paraitre mineure, mais qui l'ont influencé d'une manière qui, pour être moins visible, n'en est pas moins profonde.

\footnotetext{
1 Cf. mon article «le film en costumes : un bon objet ?", CinemAction, n65, "Cinéma et Histoire, autour de Marc Ferro ", $4^{\circ}$ trim. 1992, pp. $111-122$.
} 


\section{1 - Les traditions narratives les plus anciennes: vies de saints, miracles, chansons de geste.}

Les parentés formelles et structurelles entre les films des premiers temps et la narration médiévale ont déjà été relevées par d'autres que moi ${ }^{2}$. Disons, en simplifiant, que le cinéma prenait le relais naturel des formules traditionnelles de narration en vignettes ou en tableaux, celles de l'image d'Epinal par exemple, mais aussi de certaines formes de spectacles, qui conservaient son contact avec des modèles narratifs archaïques, qui se maintiendront jusqu'à la fin du «mode de représentation primitif " ${ }^{3}$. Autarcie du tableau, caractère "centrifuge » de l'espace de représentation, non - linéarité et non - clôture du récit, chevauchements et répétitions éventuelles, telles sont quelques unes des caractéristiques de ce mode de représentation. Il offre bien des parentés avec le récit iconique médiéval, celui des vitraux ou des miniatures. Mais aussi, plus subtilement, la structure de ces films en tableaux évoque des formes comme les séquences liturgiques ou les laisses épiques. Les exemples les plus purs en sont fournis par les nombreuses Passions et vies $d u$ Christ qui prolifèrent dans le cinéma des premiers temps. ${ }^{4}$ Il est frappant de noter que la narration cinématographique commence par là même où avait commencé la narration écrite occidentale. Je n'irai pas jusqu'à dire que la Passion Pathé ou celle de Jasset doivent quelque chose à celle d'Arnoul Gréban ou de Jean Michel ; mais elles en descendent en ligne indirecte, à travers de multiples médiations. Dans la même voie, le cinéma des premiers temps s'est essayé à un genre originel, celui des vies de saints. ${ }^{5}$ Il est vrai que peu de ces saints sont des saints médiévaux. Mais le modèle qui encode la narration en tableaux, lui, doit beaucoup à une tradition inscrite dans la longue durée. Aussi, des vies de saints postérieurs au Moyen Age seront présentées dans un récit de type «moyenâgeux ». J'entends par là, d'abord, la condensation qui se produit dans certains films, en un seul plan, celui-ci suffisant à évoquer l'épisode-clé de la vie du saint, qui représente, en quelque sorte, son attribut fondamental. La Tentation de saint Antoine illustré par Méliès en 1899 et par Lumière en 1900 sont ainsi constituées. Il est vrai que la première se distingue par son irrespect : le Christ en croix au pied duquel le saint essaie de retrouver quelque calme se transforme en jolie jeune femme aguichante!.. On est ici plus proche de Flaubert et de Félicien Rops que de la Légende Dorée. Dès les origines, le cinéma marque ainsi sa liberté de jouer avec les thèmes transmis par la tradition.

\footnotetext{
${ }^{2}$ Tom Gunning, "Le Style non continu dans le cinéma des premiers temps ", Les Cahiers de la cinémathèque, Perpignan, $n^{\circ} 29,1979, p p .2434$. François de la Bretèque, «Le Moyen Âge dans le cinéma des premiers temps ", Cahiers de Conques, à paraitre.

${ }^{3}$ Noël Burch, « Un Mode de représentation primitif ? ", La Lucame de l'Infini, Nathan, 1990, pp. $173-$ 190.

${ }^{4}$ Noël Burch, "Passions, poursuites, d'une certaine linéarisation", ibid. pp. 137-155 ; "Pierre Leprohon, "Les évocations directes de la Passion, du début du cinéma jusqu'en 1939 ", Études cinématographiques $\mathrm{n}^{\circ} 10 / 11$, automne 1961, pp. 135-149; "La Passion filmée, questions de narration ", dans Une Invention du diable ? Cinéma des premiers temps et religion, coll. sous la dir. de Roland Cosandey,'André Gaudreault et Tom Gunning, Presses de l'Univ. Laval et Ed. Payot, Lausanne, 1992, pp. 91-144.

${ }^{5}$ François de la Bretèque, «Les films hagiographiques dans le cinéma des premiers temps », op. cit. pp $121-130$.
} 
Lorsque le film comporte plusieurs tableaux, le mème phénomène de condensation et d'emblématisation peut s'observer. Ainsi, dans les quelques bandes consacrées aux martyrs: Les Martyrs chrétiens (Nonguet, Pathé, 1905) ou Aux Lions les chrétiens (Feuillade, Gaumont, 1911), les tableaux se succèdent dans un ordre plus énumératif que diégétique. Le "désamorçage du narratif », dont Alain Boureau fait une caractéristique de la Légende Dorée, s'observe même dans les biographies de saints plus historiques, comme Jeanne d'Arc ou François d'Assise. On n'a ni le temps, ni la volonté d'évoquer le processus qui conduit leur destin. ${ }^{6}$. Avec la discontinuité, la frontalité de la mise en scène et l'adresse fréquente aux spectateurs achèvent de donner à ces films leur style singulier, qui est moins dépendant d'une esthétique théâtrale, comme on le dit, que des formes les plus traditionnelles de la narration iconique.

Proches des vies de saints, se trouvent les récits de miracles. Le cinéma naissant redécouvrait la question des limites du représentable. Instrument fascinant, il révéla vite à ses utilisateurs qu'il leur permettait de manipuler les apparences et de donner corps à l'invisible. Les anges et les diables proliférèrent donc volontiers dans les premiers films, dont les auteurs étaient parfois, comme Méliès, d'anciens illusionnistes. ${ }^{7}$ Dans son souci de respectabilité, et par proximité avec la culture de la partie populaire de son public, le cinéma se mit, pendant toute une période, à multiplier les récits de miracles, notamment des miracles de la Vierge. La Légende de la fileuse, Le Rêve de la dentellière, La Légende du point d'Argentan, La Légende du sonneur, s'ils ne se déroulent pas tous explicitement dans un cadre médiéval, doivent tous quelque chose aux recueils médiévaux tels que les avait transmis la Bibliothèque Bleue. ${ }^{8}$ Jacques de Baroncelli ne déclare-t-il pas, en 1923 son intention de réaliser une adaptation du Tombeur de Notre Dame ? $^{9}$

Le Rêve de la dentellière (film Lux, 1905) présente, dans un univers moyenâgeux, une pauvre jeune fille, épuisée, qui s'endort sur son travail. La Vierge apparaît alors et termine la dentelle à sa place, puis l'apporte au château et se fait grassement payer; elle rapporte l'argent avant de s'éclipser discrètement. Dans tous ces récits, certes, le sacré s'est bien évaporé. Seul reste l'émerveillement suscité par les possibilités de trucage, et l'affranchissement des lois de la réalité que le nouveau moyen d'expression permet. Mais cet effet, qui n'était pas absent des récits médiévaux, persistera au-delà de la période primitive. Baroncelli, déjà cité, adapte en 1923 un conte de Nodier, qui avait été repris par Maeterlinck, avant de séduire Max Reinhardt en 1913. ${ }^{10}$ Cette Légende de Saur Béatrix, il déclare l'avoir tirée de la compilation d'Hugues Farsit (?) et de Gautier de Coincy. Il la résume ainsi : « La Vierge vient remplacer la sacristine d'un couvent, qui s'est enfuie pour vivre dans la

\footnotetext{
${ }^{6}$ Il faut rappeler que jusque vers 1910 , un bonimenteur se trouvait souvent dans la salle pour commenter l'action et palier les lacunes. Sa fonction sera rendue inutile par l'apparition des intertitres.

${ }^{7}$ Il y a une étude à faire sur Méliès et l'iconographie médiévale. Il est certain qu'il doit quelque chose aux formes du théâtre médiéval dans certaines de ses féeries. Je renvoie au chapitre sur Méliès de ma thèse «L'Imaginaire médiéval dans le cinéma occidental ».

${ }^{8}$ Il faudrait y ajouter des contes comme Geneviève de Brabant ou Robert le Diable.

9 Jacques de Baroncelli, Comoedia, $1^{\circ}$ juin 1923.

${ }^{10} \mathrm{Le}$ grand metteur en scène allemand en fit une pièce de thêâtre, puis un film, aujourd 'hui perdu. cf. Bradford Smith, «A Religious Spectacle in Theater and Film : The Miracle (1911-1912), Cinéma des premiers temps et et religion, op. cit. pp. 311-318. Avant Reinhardt, Michel Carré en avait filmé une adaptation en 1912 .
} 
débauche, en sorte qu'on n'a pas remarqué son absence lorsqu'après bien des années le repentir la ramène ». "Sans le vouloir, peut-être, confie-t-il, j'ai cédé à la mode : Le Moyen Age, un Moyen Age dégagé de ces fameuses ténèbres qu'épaississait, naguère, toute une tradition oratoire et politique, retrouve sur nos esprits son crédit et sa séduction ". ${ }^{11} \mathrm{Ce}$ Moyen Age des miracles, forme encore le clou du Miracle des Loups ; (1924) ; et, quoique fort éloigné des sources médiévales authentiques, Les Visiteurs du soir, (1942),accusés par les médiévistes de propager une image frelatée du Moyen Age, ne repose-t-il pas sur le même émerveillement devant les apparitions et substitutions?

Le cinéma oubliera vite, pourtant, les vies de saints. Il faudra attendre 1992 et le film étrange de Serge Roulet, Le Voyage étranger, pour retrouver une incursion audacieuse dans ce domaine. Ce film lent et méditatif se présente comme une adaptation de La Vie de Saint Alexis. ${ }^{12}$ Bien que la tentative ne soit pas parfaitement aboutie, ce film manifeste une véritable ambition: le "voyage " en question est un voyage intérieur, celui qui conduit lentement le personnage vers la grâce. Mais le cinéma modeme a peut-être plus de difficultés que celui des origines à se couler spontanément dans les moules narratifs de notre littérature naissante.

En France, il ne réussira guère non plus à retrouver les voies de la chanson de geste. On le dit, les Américains y parviennent beaucoup mieux que nous...Le western ne constitue-t-il pas leur épopée de fondation et leur Moyen Age, comme le veut un cliché répandu ? ${ }^{13} \mathrm{Ne}$ répète-t-on pas aussi, à satiété, que les Français n'ont décidément, au cinéma comme ailleurs, pas la tête épique?

Tout en laissant en friches la plus grande partie de l'héritage littéraire en ce domaine (personne n'a songé à tirer un film de la Geste de Guillaume ou de celle de Renaud de Montauban), le cinéma français s'est quand même mesuré à Roland. Ce héros ne lui réussit guère, peut-être parce que les Italiens s'en étant emparés depuis longtemps, ils sont mieux à même de rendre le caractère baroque qui est désormais attaché à son image. Les Français firent une tentative, dans le contexte du «Film d'Art», cette petite révolution des formes cinématographiques qui ramena le cinéma vers le théâtre, les décors et les sujets « sérieux ». De plus, vers 1910, Roland avait tout pour foumir un héros à la propagande patriotique et nationaliste. ${ }^{14}$ C'est Louis Feuillade, directeur artistique très catholique de la maison Gaumont, qui réalisa ce Roland $\dot{a}$ Roncevaux. en juin 1913 Il se présente comme une «chanson de geste en 8 tableaux ». C'est un habile panaché de La Chanson de Roland et du

\footnotetext{
${ }^{11}$ Loc. cit Le miracle de la sacristine n'en avait pas fini avec le cinéma : Buñuel le raconte dans la Voie lactée, par la bouche du curé...

${ }^{12} \mathrm{Au} \mathrm{XI^{ \circ }}$ siècle, le jeune noble Alexis décide de quitter les facilités du monde. Après un séjour au couvent, il part pour l'Orient. Réduit en esclavage, il sympathise dans la prison avec un vieux musicien arabe. Il est libéré. Il revient incognito au pays et observe son fils, devenu adulte, qui se convertit après lui avoir parlé. Alexis meurt alors, réconcilié avec le monde.

${ }^{13}$ André Bazin, notamment, l'a popularisé dans son célèbre article "Évolution du western », Les Cahiers du cinéma, déc. 1955, repris dans Qu'"est-ce que le cinéma ?, L. 3, p. 150. Jean A. Gili, «le western et les chansons de geste ", Études cinématographiques, 1961. Dans un même mouverment, on accuse les Américains, quand ils touchent au Moyen Âge, de transformer celui-ci en western.

${ }^{14}$ Christian Amalvi, Le Goût du Moyen Âge, Plon, 1995, pp. 231-232. Le succès de la pièce d'Henri de Bornier, La Fille de Roland (1875), précéda la réédition de Léon Gautier (1882), qui souhaitait inscrire la Chanson de Roland au programme de toutes les écoles.
} 
Roland Furieux de l'Arioste. L'action est en effet resserrée autour de l'épisode de Roncevaux. Mais on a choisi d'expliquer la trahison par le dépit amoureux. Angélique, courtisée par Ganelon, préfère Roland, qui tombe sous son charme. Le guerrier dédaigné va vendre ses services à l'émir de Saragosse. La trahison obéit donc à un motif d'ordre privé, rappelant quelque peu les structures du drame bourgeois. La dimension politique du thème n'est présente qu'en creux.

Roland sera oublié par le cinéma français, jusqu'au film de Frank Cassenti (La Chanson de Roland, 1977) qui porte, à son tour, la marque de son époque de réalisation. Non sans mérite, il choisit d'exposer les conditions de création de la chanson de geste, sur la route des pèlerinages au XX' siècle. C'est un thème que le cinéma n'avait jamais abordé jusqu'alors et qui ne fut pas repris. La lecture nationale, voire nationaliste, qui avait prévalu dans les premières années du siècle, laisse la place à une vision plus politisée et plus internationaliste. Fortement influencé par la vulgate marxiste, le film de Cassenti montre la création de la chanson de geste comme la diffusion d'une sorte d' " opium du peuple» par les seigneurs et par l'Église, pour détourner vers l'imaginaire les velléités de révolte des pauvres gens qui forment la masse des pèlerins. Il doit plus à Brecht et à sa conception du théâtre "épique » qu'à la tradition de la chanson de geste. Celle-ci semble être restée inaccessible au cinéma français. Les véritables pages épiques de celui-ci-car elles existent - ne se rencontrent pas dans les adaptations directes, mais dans des spectacles dont la caution culturelle est moindre: les superbes scènes de bataille du premier Miracle des Loups, (Raymond Bernard, 1924), par exemple.

\section{2- La littérature romanesque.}

Contrairement à une idée reçue, le cinéma, même à ses débuts, s'est bien davantage inspiré du mode de narration du roman que de celui du thêâtre. Mais, à mesure qu'il s'institutionnalise, le modèle romanesque qui s'impose est celui du roman réaliste-naturaliste du XIX ${ }^{e}$ siècle. Les particularités de la narration médiévale, dont nous avons dit que, au départ, elles n'étaient pas si éloignées de celle du cinéma primitif, deviennent peu à peu incompréhensibles ou inutilisables. Si bien que l'on assiste à ce paradoxe : pour adapter un récit médiéval, le cinéma classique le fera transiter par une réélaboration selon ce modèle moderne. C'est tout le travail du cinéma hollywoodien (où le roman scottien remplit cette fonction médiatrice). En France, cette procédure ne rencontrera pas le même succès, elle se heurtera à des réticences ${ }^{15}$. C'est pourquoi, sans doute, les adaptations directes de textes médiévaux ne se rencontrent guère qu'en deux périodes, séparées par un grand vide : avant 1914, et après 1970 .

\footnotetext{
${ }^{15}$ On peut proposer de celles-ci des explications multiples. Il est certain, $d$ 'abord, que l'accès au corpus médiéval est moins direct en France que dans les pays anglo-saxons. Ceux-ci lisent Malory et Chaucer, nous ne lisions pas Chrétien de Troyes. D'autre part, il ne se trouve pas en France de grandes figures médiatrices, comme Tennyson, ou T.H. White, ou encore Wagner. Le respect, pense-t-on, a paralysé la France dans son rapport à son héritage médiéval. Ces généralités demandent à être nuancées. En particulier, il faut périodiser cette appréciation : avant 1914, on a «moins de complexes »; après 1940, se produit un premier «retour au Moyen Âge» (à ses textes), comme il y en aura un, mieux connu, après 1970, quand l'École des Annales débouchera sur la scène médiatique.
} 
La matière qui a eu le succès le plus constant (même si discontinu) est celle de Tristan. En France, par-đisssus la célébrité de l'opéra de Wagner, l'adaptation de Joseph Bédier, parue en 1900, contribua beaucoup à asseoir sa popularité. Deux films dans les années 10 , un au début des années 20, un dans les années 40: mais, après l'Étemel retour, suit un silence de trente ans. Il faut attendre ensuite les années 1973 à 1988 pour voir réapparaître le mythe sur l'écran. En 1911, Albert Capellani touma un Tristan et Yseut pour le compte de la SCAGL $^{16}$, film aujourd'hui disparu. La même année, Ugo Falena récidive pour la Film d'Arte Italiana ${ }^{17}$ Le roman est condensé en une vingtaine de tableaux, qui comportent les «passages obligés", mais également des variantes sur la trame de base. Brengain est amoureuse de Tristan, elle veut empoisonner les amants et c'est par erreur qu'elle leur présente le philtre. Elle dénonce ensuite les amants au roi Marc, qui est ici un vieillard magnanime. Mais ceux-ci choisissent pour finir la voie du suicide. En 1920, un grand film de prestige aujourd'hui bien oublié est produit par les studios Nalpas, sur un scénario de Frantz Toussaint et Jean Louis Bouquet. Ce Tristan et Iseult ${ }^{18}$ est divisé en six chants, qui suivent avec une assez grande fidélité la trame du récit de Bédier. Le tournage sur la Côte d'Azur eut quelque peine à trouver l'atmosphère susceptible d'évoquer les brumes du Morois. «Les brouillards atlantiques se rencontrent peu sur les bords de la mer latine. Le film, louable par son intention de grandeur, manquait du souffle indispensable à l'expression de l'ardeur désespérée des deux grands amants légendaires, Certains sujets se hérissent d'intransigeance et requièrent l'exaltation $\gg .{ }^{19}$

Il faut sauter ensuite jusqu'en 1943, pour retrouver, avec l'Etemel Retour, Tristan et Yseut sur les écrans français. Je n'aurai pas la prétention d'étudier ce film célèbre (et injustement décrié) dans le cadre restreint de cet article. ${ }^{20} \mathrm{La}$ «modemisation diégétique ${ }^{21}$ à laquelle s'est livrée le poète, en changeant les noms, le cadre, l'époque, tout en conservant le fond, a beaucoup été commentée. Il faut y reconnaître, outre les préoccupation propres à l'univers et à la philosophie de Cocteau, une sorte de marque de timidité, comme si l'époque hésitait encore à traiter directement du Moyen Age, et éprouvait le besoin de le «déguiser » pour le rendre acceptable.

Les deux plus récentes adaptations cinématographiques de Tristan en France, celle de Yvan Lagrange et celle de Louis Grospierre (Connemara) ont adopté d'autres partis. Lagrange déconstruit complètement la narration et livre au spectateur une structure très originale, plus poétique que narrative. Il serait vain d'essayer d'y reconnaître l'enchaînement des épisodes de la légende de Tristan. Ils sont traités en motifs poétiques, ou symphoniques,

\footnotetext{
${ }^{16}$ Société Cinématographique des Auteurs et Gens de Lettres, filiale de Pathé, créée dans l'intention de contrôler les droits d'exploitation du patrimoine littéraire.

${ }^{17}$ En dépit de son nom, il s'agit d 'une société à capitaux français, dépendant encore de Pathé. Peut-on pour autant dire qu'il s'agit d 'un film français ? Les interprètes, dont Francesca Bertini, le réalisateur, les décors sont italiens...L 'historien est incité à la prudence.

${ }^{18}$ Aujourd 'hui invisible, mais conservé au Service des Archives du Film (copie nitrate non restaurée).

${ }^{19}$ Henri Fescourt, La Foi et les montagnes, ed. Paul Montel, 1959, p. 193.

${ }^{20}$ Voir par ex. Loetitia Papon, $L$ 'Envers et l'Endmit dans l'Étemel Retour de Jean Cocteau, mémoire de maîtrise, Université Toulouse-Le Mirail, 1995 ; Bulletin de la Société des Amis de Jean Cocteau, $n^{\circ}+25$ et 26,1995 . Je me permets de renvoyer à nouveau aux deux chapitres de ma thèse consacrés aux " anachronismes » et à «Tristan ».

${ }^{21}$ Expression de Gérard Genette dans Palimpsestes, la littérature au second degré, Seuil, 1982.
} 
mais néanmoins parfaitement reconnaissables. Le film entrelace les deux thèmes du couple, fragile, enlacé sous des peaux de bêtes précaires posées à même les galets de la plage, et des combats titanesques entre des chevaliers lourdement cuirassés. Tristan en revient toujours blessé, saignant par la bouche, la poitrine. Iseult le soigne, le conduit à l'écart, lui fait vivre des moments d'extase hors de cette fureur guerrière. Le jeune cinéaste a réduit le mythe à une épure. Il a spontanément retrouvé, par des voies détoumées, une construction lyrique, incantatoire, qui retrouve quelque chose de l' «archaïsme » du roman de Béroul. II y a moins à dire du film de Grospierre, qui propose une version "barbare" dans laquelle les personnages sont des bergers/guerriers, et où triomphe la violence décorative de l'heroïc fantasy.

De Capellani à Louis Grospierre, on mesure une régression frappante dans un cadre de plus en plus «barbare » affectant aussi bien les décors et les accessoires que la psychologie des personnages. La légende retourne à ses origines celtiques. Après les extérieurs méditerranéens du début du siècle, puis les bords du lac Léman de Delannoy/Cocteau, les œuvres les plus récentes remontent en Irlande, voire en Islande. Les premiers films tentaient bien de conférer aux costumes et aux décors une atmosphère archéo-médiévale, mais c'était encore un Moyen Age de convention. Le film de Maurice Mariaud optait pour des armures des XI'-XII ${ }^{\mathbf{e}}$ siècles, et plaçait ses personnages dans des décors monumentaux inspirés par les Niebelungen de Fritz Lang. Mais, après le détour par un $\mathrm{XX}^{e}$ siècle prosaïque dans L'Étemel Retour, il n'y a plus de palais ni de château. Les derniers films nous placent dans un cadre résolument primitif, précaire, viking, des plages nues battues par les vents, des tentes en cuir, des masures en pierre et en torchis.

Comment faut-il interpréter cette régression? Le souci de réalisme historique (les héros de la légende sont censés avoir vécu aux temps arthuriens, soit au vie siècle) ne constitue qu'une détermination de surface. C'est, en réalité, le noyau sémique du mythe lui-même qui est affecté par cette translation. C'est notre vision de l'amour, du couple, de la guerre, des relations de pouvoir, qui sont devenues, au long du $\mathrm{Xx}^{e}$ siècle, de plus en plus «barbares ».

La matière arthurienne, qui a été et reste le terrain favori des cinéastes américains, a, quant à elle, particulièrement intimidé les Français. On pourrait croire qu'ils ont abandonné, jusqu'à une date récente, cette mythologie à leurs vieux ennemis. L'examen des scénarios déposés à la Bibliothèque nationale m'a convaincu, néanmoins, qu'on y avait pensé, autour de 1910. Eugène Creissel proposa, en effet, à la maison Pathé, un scénario dont le personnage principal était Merlin. Il tenait à en faire une «légende celtique ${ }^{22}$ Le film ne semble pas avoir été tourné, mais il est étrange de voir un scénariste français anticiper de près de 70 ans l'Excalibur de John Boorman.

Cette tentative demeura sans lendemain. Le roi Arthur, la Table Ronde, Lancelot, Perceval, le Graal, sont demeurés très longtemps un sujet inconnu du grand public français. Etrange lacune qu'il faut certainement imputer à des raisons structurelles de l'imaginaire national. Le monde arthurien ne fait pas partie de la mythologie politique des Français. ${ }^{23}$ Une surdétermination s'y est ajoutée, vraisemblablement, qui tient au statut prestigieux dont

\footnotetext{
${ }^{22}$ Je renvoie encore à ma thèse, où j'ai étudié en détails ce scénario.

${ }^{23}$ En outre, c "est un roi ; or, en dehors de Henri IV, peu de monarques ont eu la faveur de figurer dans le Panthéon national de la République. Arthur, du reste, ne figure absolument pas dans la galerie des grandes figures transmises par l'école, et qui coïncide largement avec l'imaginaire cinématographique.
} 
jouit chez nous l'objet littéraire. ${ }^{24}$ En ce domaine, il n'a pas existé chez nous de médiation comparable aux romans de Walter Scott, ou de T.H. White. C'est par conséquent au moment où l'on « reviendra aux textes», selon la formule consacrée, que l'on commencera à penser à la légende arthurienne pour un scénario de film. En réalité, j'espère l'avoir fait comprendre, on n'a pas attendu les années 1970 pour connaitre les textes; il faut bien supposer que d'autres raisons ont rendu possible leur traitement scénaristique. L'une d'entre elles est la percée de la «nouvelle histoire » et le regain d'intérêt pour les sciences historiques après $1968^{25}$; une autre est l'affirmation renouvelée du cinéma « d'auteur ». Robert Bresson, avec son Lancelot du Lac en 1974, puis Eric Rohmer, avec son Perceval le Gallois en 1978, proposèrent à peu de distance les deux premiers vrais films arthuriens français.

Il est assez tentant (sans entrer dans le détail ici non plus, faute de place) de les considérer en diptyque, car ils constituent deux réponses au défi de l'adaptation à l'écran d'un texte médiéval. Bresson s'est inspiré de La Mort le Roi Artu, bien qu'il ne le dise pas explicitement et prétende s'être inspiré assez librement d'une idée vague de la légende. ${ }^{26} \mathrm{La}$ démarche de Rohmer est différente : il affiche la fidélité à sa source, le Conte du Graal de Chrétien de Troyes, dont il donne à entendre le texte, traduit par lui-même et alternativement chanté par un choeur et récité par les protagonistes du film. La démarche hyperarchéologique de Rohmer n'est pourtant pas forcément la plus fidèle «en esprit» aux textes médiévaux... Les systèmes scénographiques adoptés par les deux réalisateurs, qui se sont l'un et l'autre posés le problème de la transcription de la façon dont les hommes du Moyen Age percevaient le monde, sont eux aussi en contraste. Chacun correspond sans doute à des choix d'auteur. Bresson a opté pour un espace contracté, abstrait, surdécoupé, qui est amplifié par une bande sonore très travaillée. Il a pratiqué par le montage tout un système d'ellipses et d'effets incantatoires de répétition: son tournoi est resté célèbre à cet égard. Rohmer a tenté une scénographie inspirée directement du théâtre médiéval et a reconstitué sur son plateau la scène «en rond». On se trouve face à deux formules radicalement antithétiques, l'une poussant à l'extrême le cinéma "de montage», l'autre optant pour la négation de l'espace tridimensionnel du cinéma. Ce qui frappe, c'est que chacun retrouve, à sa manière, les voies de l'esthétique des origines. L'espace du film de Rohmer offre des parentés avec l'espace «primitif», que nous avons décrit ci-dessus; quant à Bresson, son refus de l'identification et son travail sur les rimes du réel retrouve quelque chose de la poétique narrative médiévale, et, quoique fort éloigné du cinéma des premiers temps, son écriture se présente comme une réaction radicale contre le «mode de représentation institutionnel» qui a triomphé dans le cinéma international (américain en premier lieu) depuis 1914. Enfin, chacun présente sa propre lecture du mythe, dont les présupposés

${ }^{24}$ C'est 1 'hypothèse de Jeff Rider, Richard Hall et Christopher Smith, "The Arthurian Legend in French Cinema ", dans Kevin J. Harty, Cinema Arthuriana, Essays on Arthurian Films, Garland pub. inc., London \& New York, 1991. (Je m'en tiendrai à cette seule référence, la bibliographie sur le sujet.étant très abondante)

${ }_{25}$ Cf. mon article : "Le Moyen Âge du cinéma français, 1940 - 1987 ", dans Dire le Moyen Âge, Actes du colloque de 1987, université de Picardie, dir. p. Michel Perrin, PUF, 1990.

${ }_{26}^{26}$ J'aime mieux, comme pour ce film-ci, inventer des personnages légérement différents de ceux d'une légende assez vague et les faire parler moi-même, plutôt que d'avoir à être fidèle à des écrivains, même admirables » : propos recueillis par Yvonne Baby, Le Monde 26 sept. 1974. 
éclatent aux yeux dans la fin de chaque film. Bresson pousse le pessimisme nihiliste bien audelà de ce qu'on peut lire dans sa source supposée. Le naufrage du monde arthurien est présenté comme la métaphore du naufrage vers lequel se dirige le nôtre, à partir du moment où il a renoncé à toute dimension spirituelle. Rohmer conclut le texte inachevé de Chrétien, en imaginant que Perceval devient protagoniste d'un Jeu de la Passion. ${ }^{27}$ Il infléchit la christianisation du mythe, à l'exemple du travail auquel s'étaient livrés les continuateurs du poète champenois au XIII' siècle.

On peut dire que le passage par l'œuvre médiévale amène le cinéma sur certaines des voies les plus radicales de la modernité.

\section{3 - Les traditions anti-courtoises.}

A lire ce qui précède, on pourrait penser que, dans l'esprit des gens de cinéma en France, le Moyen Age se résume à la religion, la chevalerie et la courtoisie. Une image un peu compassée, un peu académique, qui a pu être encouragée par les milieux conservateurs, très puissants dans le monde du cinéma, et influencée par des institutions comme l'école. Mais là encore, il faut nuancer cette appréciation. Le cinéma des premiers temps foumit un nouveau témoignage précieux à cet égard. Les " petits genres" dérivés de la littérature médiévale y trouveront un lieu d'épanouissement, plus favorable que dans les époques ultérieures. Pourquoi? D'abord parce que c'était une phase d'invention et d'exploration. Sans doute aussi, parce que les barrières de la censure sociale, avant 1908, étaient encore basses et admettaient des transgressions vers l'irrationnel, le trivial, voire le scatologique. Après la période "primitive ", cette inspiration restera cantonnée dans des secteurs marginaux, ou se diluera dans l'ensemble de la production, au point qu'il devient hasardeux de l'identifier.

Le Roman de Renart en fournit un bon exemple. ${ }^{28}$ Le cinéma français, comme les autres, a fait preuve d'une surprenante timidité face à ce superbe matériau narratif (alors que les fulms animaliers sont innombrables). La seule véritable exception est constituée par un film réalisé dans des conditions strictement artisanales, le remarquable Roman de Renand de Starevitch, dont la fabrication s'est étalée de 1926 à 1941. Son auteur, polonais de Russie exilé en France, écrivit le scénario avec sa femme, en mêlant les traditions littéraires. I] fabriqua lui-même les poupées animées qui sont les personnages du film, dont il tint la caméra. Il donna une version personnelle de l'histoire en se servant à la fois du Reineke Fuchs de Goethe, du Reinhandt Fuchs médiéval, et en ajoutant des apports originaux. II prend assez franchement le parti de Renard, mauvais garçon réfractaire à l'ordre social. II transmet une jouissance ludique, voire un peu sadique, à montrer ses victimes. Il propose un discours discrètement subversif qui n'est pas sans signification au moment de la montée des totalitarismes. Le film s'achève par un triomphe ambigu du trompeur, dont le Lion décide de faire son conseiller après sa victoire dans le siège de Maupertuis. Le film de Starevitch resta longtemps invisible. Le cinéma français oublia ensuite presque complètement le Roman de

\footnotetext{
${ }^{27}$ Rohmer renoue ainsi avec la production des Passions dans le cinéma des origines, cf. ci-dessus : c'est un autre point de rencontre.

${ }^{28}$ François de la Bretèque, "Renart au cinéma, un rendez-vous manqué ", dans Le Renard, sous la dir. de Claude Rivals, à paraître ; "Renart, ou le jeu avec les lois", dans «Et c'est la fin pourquoi sommes ensemble ", Hommage à Jean Dufoumet, Honoré Champion, 1993, L1, pp. 75-88.
} 
Renart, excepté dans des dessins animés de court métrage. La matière renardienne s'y trouve décomposée en petites unités, tirant l'épopée animale dans le sens de la fable, de l'apologue. C'est précisément sous cette forme éclatée que la présence de l'intertexte renardien se laisse lire dans certaines œuvres, parfois sans rapport direct avec l'œuvre médiévale: un film comme Goupi Mains Rouges (Jacques Becker, 1942, d'après le roman de Pierre Véry) pratique une redistribution subtile des éléments signifiants du personnage de Renart, à travers la famille des Goupi (leur nom le dit clairement). Le héros, interprété par Fernand Ledoux, l'incarne un peu plus que les autres. Ce personnage s'avère positif, en dépit des premières apparences. Ce film s'inscrit, de la sorte, sur la longue durée, comme une étape de la réhabilitation de Renart ${ }^{29}$

Le cinéma primitif a puisé dans la masse des récits à "formes brèves »: contes, anecdotes, fables, dans laquelle il est possible de reconnaître, ici ou là, des récits d'origine médiévale. En 1910, par exemple, Feuillade toume un Robert le Diable - qui doit sans doute quelque chose à l'opéra de Meyerbeer ; En 1911, chez Gaumont, est déposé un scénario de Aucassin et Nicolette, prouvant que l'on connaissait déjà suffisamment la chantefable pour envisager d'en faire un film à succès (celui-ci n'a pas été tourné). ${ }^{30}$ Plus surprenant encore : la société Éclair produit, en 1912, une adaptation du Lai de l'ombre de Jean Renart, sous le titre L'Ombre de l'aimée. ${ }^{31}$ Le cinéma d'avant 1914 s'est donc davantage intéressé à cette part de l'héritage littéraire médiéval que les périodes suivantes.

Il reste un domaine dans lequel l'influence de la littérature médiévale peut se deviner, où elle a sans doute été déterminante, quoiqu'elle se soit effectuée à travers un grand nombre de médiations : c'est celui des fabliaux et des farces. Pour évaluer cette dette, il faudrait plonger dans l'énorme masse de ce que nous appelons aujourd'hui le «cinéma burlesque ». Celui-ci, à ma connaissance, n'a pas livré de transposition directe d'un fabliau ou d'une farce médiévaux; en effet, par principe, le cadre de l'action en est toujours contemporain. Cependant, la résurgence de motifs, plus ou moins autonomes, est parfois aisément reconnaissable. Cette observation avait déjà effleuré l'esprit d'André Bazin, qui écrivait, en 1951 : «Entre 1905 et 1920, la farce a connu un éclat unique dans son histoire; Je dis bien la farce, telle que la tradition s'en est perpétuée depuis Plaute...Un exemple : le thème classique du Cuvier se retrouve spontanément dans un vieux Max Linder, où l'on peut voir le sémillant Don Juan séducteur d'une teinturière, obligé de se plonger dans une cuve pleine de couleur pour échapper à la vindicte du cocu $»{ }^{32}$ Je prendrai un autre exemple : dans un film Pathé de 1905, Petits voleurs de pommes, les gamins, poursuivis par le paysan spolié, s'enfuient par un trou de la clôture, tandis que leur poursuivant, trop gros, y reste coincé: tel Primaut quand il a trop mangé de jambons, dans la Branche $X I V$. Le rapprochement, m'objectera-t-on, est un peu aléatoire. Forcément, puisqu'il s'agit de motifs « universels »,

\footnotetext{
${ }^{29}$ Autre exemple : dans le film de Suzanne Schiffmann, Le Moine et la Sorcière (1987), le dominicain Etienne de Bourbon commence son premier sermon par l'exemplum de la «mort feinte» du renard quand il chasse les oiseaux.

${ }^{30}$ Lotte Reiniger en réalisera un en silhouettes découpées, en 1975.

${ }^{31}$ Le scénario a été publiè dans la revue $1895, \mathrm{n}^{\circ} 12$. Le film a été réalisé et il est sorti sur les écrans.

${ }^{32}$ André Bazin, "Théâtre et cinéma ", Esprit, juin et juil. 1951, repris dans Qu"est ce que le cinéma op. cit. t. 2 p. 74. On relèvera que Bazin se trompe : son souvenir de la farce du cuvier est vague, il la confond avec Renart teinturier... Mais sa remarque n'en est pas moins fondée.
} 
qui voyagent d'un texte à l'autre depuis des siècles. C'est leur réinvestissement, massif dans cette production burlesque, qui autorise la mise en rapport (sans qu'on puisse parler d'influence au sens strict du terme). Passée cette période, le cinéma français parait avoir méprisé cette veine-là, davantage que d'autres cinématographies (voyez les Italiens... ${ }^{33}$ ). Elle connaîtra une petite résurgence dans les années 70 , avec la libéralisation des mœurs et la vague érotique. Un film comme L'Histoire très bonne et très joyeuse de Colinot-TrousseChemises (Nina Companeez, 1973) enfile des situations qui font implicitement référence aux fabliaux : un moine voyeur feint d'être aveugle pour mieux parvenir à ses fins; un jeune garçon se mêle au bain de jeunes pucelles faussement naïves qui prennent son sexe pour une couleuvre; Colinot, obligé de se travestir en femme pour échapper à un mari jaloux, se voit courtisé par celui-ci...C'est peu de choses, mais le grand contre-texte anti-chevaleresque et anti-courtois aura ainsi manifesté sa trace et rectifié l'image d'un Moyen Age trop solennel.

\section{Conclusion}

Le cinéma français du début du siècle s'est intéressé à la littérature médiévale, bien davantage que ce que l'on aurait pu croire. Cet intérêt parait confirmer l'idée que la vision française s'appuie sur une tradition de respect du patrimoine littéraire, alors que les Anglosaxons manifesteraient à son égard une plus grande désinvolture. Mais on a vu que cette inspiration a connu de longues éclipses, et que pour revenir aux textes du Moyen Age, il a fallu de longs détours. Il serait peut-être plus juste de dire que la littérature médiévale est tenue chez nous pour un objet irréductiblement éloigné, qui oblige au respect ou à une démarche savante; alors que les Américains vivent leur relation avec elle dans une familiarité plus grande, fût-ce au prix de l'irrespect. ${ }^{34}$ Toutefois, cette conclusion demande à être nuancée à son tour. La matière médiévale a fonctionné comme un vaste intertexte culturel, dans lequel le cinéma français a puisé des éléments éparpillés, parfois brouillés, le plus souvent mêlés à d'autres, mais qu'un examen un peu attentif permet souvent de reconnaître.

François de la Bretèque (Université de Montpellier III)

\footnotetext{
${ }^{33}$ Pasolini : Les Contes de Canterbury, Monicelli, L'Amata Brancaleone, par exemple.

${ }^{34}$ Ana Fernandez, "La Literaura medieval y el ciné ", dans : Literatura i cinema occidentals, dir. Pere Comellas i Antoni Dalmases, Ajuntament de Sabadell, Filmoteca de la Generalitat de Catalunya 1993.
} 